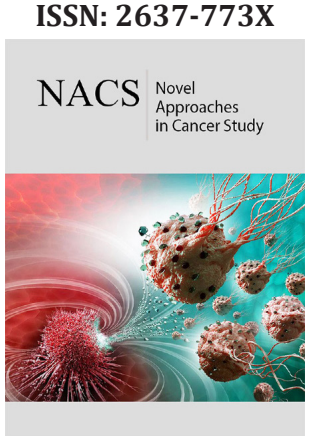

*Corresponding author: Yi Lu, Department of Pathology and Laboratory Medicine, University of Tennessee Health Science Center, Cancer Research Building, Room 258, 19 South Manassas Street, Memphis, TN 38163, USA, Email: ylu@uthsc.edu

Submission: 觜 April 01, 2020

Published: 眥 May 08, 2020

Volume 4 - Issue 4

How to cite this article: Annie Leow, Chikezie O Madu, Yi Lu. The Role of CyclinDependent Kinases on the Metastasis of Breast Cancer. 4(4). NACS.000594. 2020. DOI: 10.31031/NACS.2020.04.000594

Copyright@ Yi Lu. This article is distributed under the terms of the Creative Commons Attribution 4.0 International License, which permits unrestricted use and redistribution provided that the original author and source are credited.

\section{The Role of Cyclin-Dependent Kinases on the Metastasis of Breast Cancer}

\author{
Annie Leow ${ }^{1}$, Chikezie 0 Madu $^{2}$ and Yi Lu ${ }^{3 *}$ \\ ${ }^{1}$ Departments of Biology and Advanced Placement Biology, White Station High School,
} Memphis, TN 38117, USA, Email: andreanaleow@gmail.com

${ }^{2}$ Departments of Biology and Advanced Placement Biology, White Station High School, Memphis, TN 38117, USA, Email: maduco@scsk12.org

${ }^{3}$ Department of Pathology and Laboratory Medicine, University of Tennessee Health Science Center, Memphis, TN 38163, USA, Email: ylu@uthsc.edu

\begin{abstract}
Cyclin-Dependent kinases (CDKs) function in mitosis by allowing the cycle to progress from one stage to another due to their properties as a family of protein kinases. Because of this function, abnormalities with CDKs can lead to uncontrolled cell division, leading to diseases such as cancer. Breast cancer is one form of cancer in which CDKs are a prevalent area of study. The role CDKs play in controlling and coordinating cell division makes it an important process to understand in breast cancer and, specifically, the metastasis of breast cancer. Lack of controlled CDK function could allow the cancer to spread to other parts of the body, leading to metastasis. Inhibiting CDK activity is an area of interest in searching for ways to treat breast cancer, especially once it has spread to the point where tumors cannot be surgically removed. Investigating these pathways and the effects of CDK inhibition on breast cancer cells has revealed much on the reestablishment of cell cycle control, which consequently leads to control of the cancer. This could be an effective form of non-localized treatment against metastatic cancer that is able to target specific cells throughout the body.
\end{abstract}

\section{Introduction}

Without regulation, the cell cycle would be continuous, leading to uncontrolled mitosis. Undeterred growth in the body leads to formations of tumors, which further create complications. Fortunately, there are different checkpoints between phases of mitosis, meaning each phase does not simply occur in succession of the previous one. This is a fundamental characteristic of eukaryotes, one that has been evolutionarily conserved, showing its importance to life. One form of regulation of the cell cycle comes from CDKs, which phosphorylate certain proteins in order to drive downstream processes between the G1, S, G2, and M phases of mitosis [1]. There are different pathways for different ways of regulation. For example, entry into the M phase involves activation of M phase promoting factor (MPF), as well as inactivation of protein phosphatases [2]. These are two different mechanisms that function differently but for the same purpose.

CDKs belong to a family of serine/threonine kinases whose activity is governed by the accumulation and degradation of cyclin, a noncatalytic regulatory subunit [3]. Accumulation allows association of cyclin to the protein kinase, activating it, while degradation allows termination of the activity. The significance of such a control over the cell cycle was implicated in a study on fission yeast and cell division, where the CDK/cyclin complex Cdc2 was genetically analyzed and shown to play a part in the timing of mitosis [4]. Unsurprisingly, the deregulation of such pathways can lead to a variety of complications, including breast cancer.

A major cause of cancer is the uncontrolled proliferation of cells leading to tumor clonality, in which a single cell replicates abnormally; this can lead to both benign and malignant tumors [5]. Both types of tumors can be discussed in the context of CDKs and breast cancer. The metastasis of breast cancer would mean a malignant tumor and is much more difficult to remove locally than a benign tumor [5]. For this reason, inhibition of CDKs has emerged as a prospective way to regain control of the cell cycle. Inhibition of certain CDK pathways has led 
to arrest of cancer cells in the G1 phase of the cell cycle. An aspect of CDKs that makes this a plausible solution is their specificity. Cyclins are specific to their associated CDK, a complexity shown in how different types of cells are able to undergo mitosis at different times [6].

Breast cancer affects people, mainly although not confined to, women globally. Unfortunately, current treatments often fail due to patients developing resistance to targeted and non-targeted options. In light of this, further research on CDKs has continued, especially the CDK4/6 [7]. This particular CDK is connected to overexpression of cyclin D1. The intent of studying CDKs like CDK4/6 is to develop ways to inhibit their activity to inhibit growth of cancerous tissue while not damaging healthy tissue [7]. Furthermore, this approach to treating breast cancer poses a potential way of avoiding chemotherapy-induced alopecia (CIA), a side effect of treating cancer with chemotherapy, or cytotoxic antitumor agents. Such agents divide the epithelium of hair follicles, leading to hair loss, whereas inhibition of CDKs, specifically CDK2 inhibition, reduces the chances of this happening [8]. CDK inhibition and understanding the function of CDKs represent safer, more effective ways of treating breast cancer.

Breast cancer often spreads to places like the liver, brain, and bones, becoming metastatic. In the context of specific locations, CDK inhibition has targeted ways of reaching the areas of the body to which breast cancer spreads. For example, a common complication in breast cancer is brain metastases, to which researchers have responded by actively searching for ways through which CDK inhibitors can cross the blood brain barrier [9]. This is an example of how studying the role of CDKs in the pathology of breast cancer and the reestablishment of control of the cell cycle may yield successful results. It also allows flexibility with regards to each particular case. This review will discuss CDKs and their influence on treating metastatic breast cancer.

\section{Structure}

CDKs are essentially composed of two subparts: the kinase and the cyclin. As an enzyme, the structure of a protein kinase is highly conserved [10]. Therefore, studying the structure of a CDK yields much information about the enzyme family and its subclass. The kinases are activated by cyclin, the subparts, and crystallographic studies have allowed insight into how CDKs make the switch from inactivated to activated to inactivated again. The catalytic cleft of the kinase undergoes a conformational change with an intrinsic conformational flexibility. This flexibility enables CDKs to have a wider range of responses to different growthregulatory signals [11]. In reaction to each signal, CDKs are able to phosphorylate different proteins by adding a phosphate group to substrate proteins, leading to cell division. CDKs, as they are serine/ threonine kinases, primarily phosphorylate serine and threonine residues with their serine/threonine catalytic core [12]. Another way CDKs are able to have specificity is through the different cyclin and kinase associations. For example, there are different types of CDKs that depend on the different relationships between kinase and cyclin. CDK2 can be activated by both cyclin E and cyclin A, but each cyclin leads to a different response [13]. As shown in Figure 1, each cyclin and kinase pair acts as a checkpoint for different phases of the cell cycle, leading to a coordinated process. This shows how the structure of a particular CDK caters to its specific purposes. Studying the crystal structure of CDK2 revealed its unique helixloop segment, which was concluded to play a role in interfering with ATP and protein structure binding. This likely helps regulate other CDKs [14].

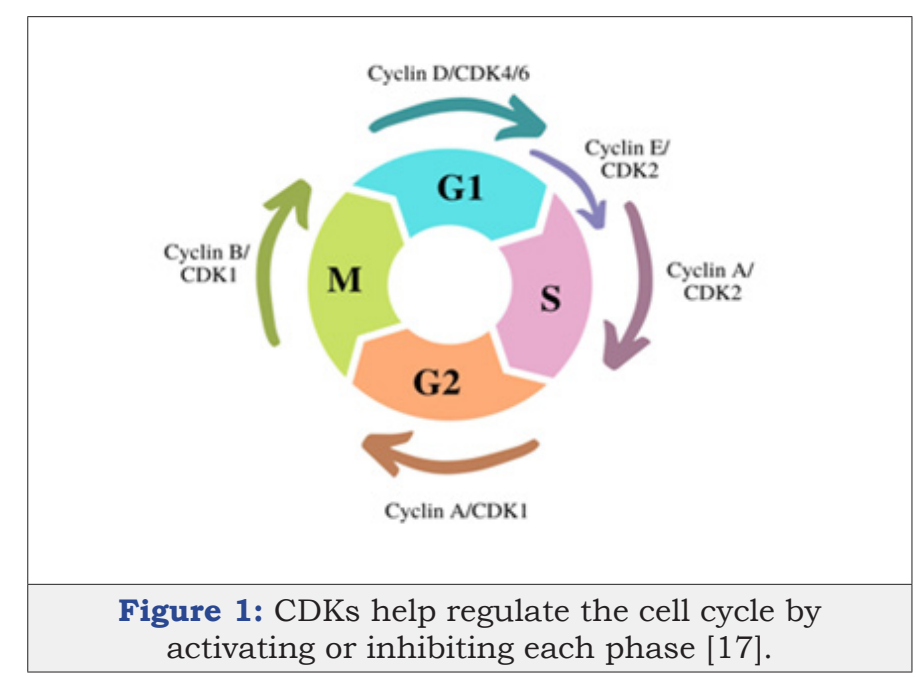

If this phosphorylation were continuous, cell division would also be continuous. Unchecked, this could wreak havoc on the body. The protein subparts, cyclins, are the key to regulating this process. The expression of cyclins is not continuous and only occurs at certain, distinct times [15]. This discontinuous expression allows for different amounts of cyclin to accumulate, which is what protein kinases respond to. When cyclin levels are high, protein kinases are activated, and the cell is able to proceed through cell division. When cyclin levels are low, protein kinases are deactivated, and the cell does not proceed through cell division. Another enzyme involved in the process is called a phosphatase. Phosphatases remove phosphate groups from proteins, allowing dephosphorylation. Similarly, cyclin degradation occurs by various enzymes, allowing the deactivation of CDKs [16].

\section{Functions and Mechanics}

CDKs most prominently function in regulation of the cell cycle $[17,18]$. They act as checkpoints for each phase of mitosis. These checkpoints monitor the size of the dividing cell, the replication of DNA, and the segregation of chromosomes during anaphase of mitosis [19]. Cyclins and CDKs work together to achieve this efficiency in the cell cycle; CDKs are able to phosphorylate and promote each phase, while cyclins are the subparts that activate CDKs. Put simpler, CDKs are the engines behind cell division, while cyclins are the gears that turn that engine [18]. Each checkpoint creates a series of dependencies, wherein the cell cycle will not proceed until a certain mitotic event occurs. For example, a study in fission yeast revealed that size is a determinant in cell division [19]. That is, when the cell reaches a certain point, it will divide. When an abnormality in cell division is detected, the checkpoint for that particular phase will be activated, and through changes in 
cyclin-CDK levels, the cell will arrest to prevent reproduction of an abnormal cell, as shown in Figure 2 in response to DNA damage [20]. The DNA replication checkpoint prevents replication of cells with damaged DNA, while the metaphase/anaphase checkpoint prevents mutations like disjunctions due to improper attachment of chromosomes to spindle fibers; the importance of such regulation means CDK pathways are highly conserved [19,20]. DNA damage can occur intrinsically, such as through misplaced spindle fibers or attrition of telomeres, or extrinsically, through sunlight or carcinogens [19]. The results of this damage left unchecked can result in cancer.

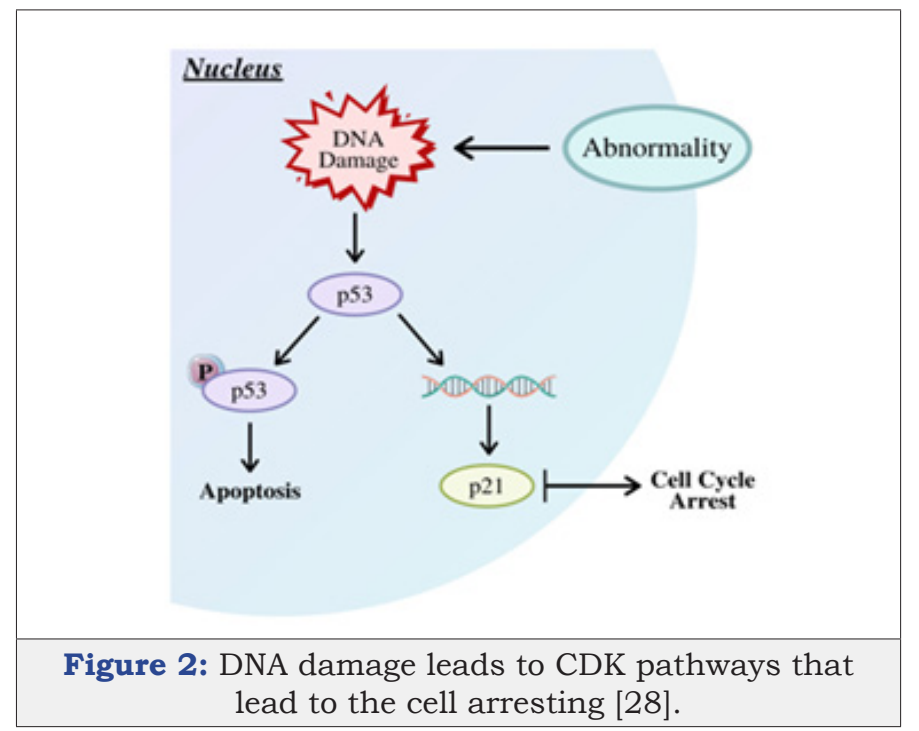

Before CDKs can regulate the cell cycle, the cell cycle must actually begin, which occurs at the restriction or $\mathrm{R}$ site as the cell enters the G1 phase. Getting from G1 to S involves more than just varying levels of cyclin and CDKs; the mechanisms through which CDKs function can vary between each phase. The G1/S transition is often considered the point at which a cell commits to dividing or not dividing. One particular mechanism involves the retinoblastoma susceptibility gene (RB). RB can be phosphorylated by CDKS, which are often enhanced or inhibited in this phase by protein kinase C (PKC) [21]. In particular, levels of CDK2 have been studied in the progression from G1 to S. CDK2 inhibition influences phosphorylation of RB and has been shown to be regulated by PKC [21,22].

Another way CDKs regulate the cell cycle is by regulating metabolic enzymes needed for certain pathways directly. These pathways are generally involved in DNA replication or other processes of the cell cycle, but this function of CDKs has come to include regulating enzymes involved in central carbon, energy, and lipid metabolism [23]. Furthermore, CDKs can also regulate these enzymes indirectly. They do this through phosphorylation of regulators of central carbon metabolism, transporters, and regulators of mitochondria [23].

Outside of the cell cycle, CDKs have been found to play important roles in organismal homeostasis. CDK4 is a known noncell cycle CDK and assists in the regulation of glucose. Although it also functions in G1, CDK4 can regulate the secretion of insulin from certain $\beta$-cells in the pancreas, and a study with mice with CDK4 defects revealed what happens without this regulation: the mice had defective pancreatic cell growth, as well as diabetes, increased glucose levels, and decreased insulin levels [23,24]. This gives insight into the importance of the dual purpose of CDK4 and potentially other CDKs. Cyclin-CDK complexes have also been found to play a role in controlling transcription and post-transcriptional modifications such as RNA splicing [25].

CDK and checkpoints are essential for regulating the cell cycle as a whole, especially to ensure that cell division actually proceeds throughout the cycle, but the specific timing of each phase is also important to consider. S phase CDKs, such as the previously discussed CDK2, ensure that DNA replication occurs only once throughout each cycle [26]. This prevents any cell, other than those in the G1 phase, from replicating DNA. Mitosis is promoted when cyclin B accumulates, activating $\mathrm{M}$ phase CDKs. This only occurs after the S phase is complete [26]. This coordinated step-by-step process of the cell cycle prevents abnormalities, such as having too much or too little DNA, from replicating.

An essential step in CDK pathways involves the dephosphorylation and inactivation of cyclin-CDK complexes. The entire mechanism of such a complex includes binding of a cyclin to a CDK, removal of inhibitory phosphorylations, and the addition of activating phosphorylations. The cyclins are cyclically transcribed, synthesized, and degraded [27]. This process results in varying levels of cyclin, even as levels of CDK remain relatively stable, allowing CDK activity to be discontinuous and cyclic, which establishes the start and stop of each phase of the cell cycle. Without this aspect of cyclin-CDK complexes, there would likely not be distinct phases or checkpoints [28].

\section{Role in Breast Cancer}

Breast cancer is an extremely prevalent form of cancer, especially in women, affecting about one in eight women in the US [29]. Among the pathways regulating normal breast development, such as estrogen receptor pathways and HER2 pathways, are CDKs and CDK inhibitors. CDK inhibitors arrest cells in cancerlike proliferation preventing the development of breast cancer [30]. This is the characteristics of CDK inhibitors that researchers primarily focus on when examining their connection to the cell cycle in the context of the development and treatment of breast cancer. Because of this aspect of CDK regulation in the role of maintaining normal cell division, the pathology of breast cancer can be tied to abnormalities in CDK pathways.

Because of their ties with the development of breast cancer, CDKs can also be used to help treat breast cancer by using CDK inhibitors to regulate proliferation. For example, breast cancer can be hormone-receptor positive or hormone receptor negative, depending on the presence of hormone receptors in breast cancer cells that have been biopsied. This is important to know when treating breast cancer because hormone receptors, such as estrogen receptors, because these receptors fuel growth of cancer cells [31]. 
It has been shown that selective CDK4/6 inhibitors can be used to treat estrogen receptor-positive breast cancers because they block the growth of such receptors, thus helping stop the growth of cancer cells [29]. CDK4/6 interact with cyclin D to play an active role in DNA replication and, therefore, a cell's progression through the cell cycle [32]. In the absence of such regulation, breast cancer could develop as cells divide uncontrollably.

In other cases, CCND1, the oncogene that encodes cyclin D1, behaves abnormally, leading to overexpression of cyclin D1, which oversees transition from G1 to S [33]. Because the inactivation of CDKs involves falling levels of cyclin, this would create a lack of regulation in CDKs, leading to an uncontrolled cell cycle. Cyclin D1 is closely associated with breast cancer because it is overexpressed in a high number of cases. The overexpression of estrogen receptor $\alpha(E R \alpha)$ is correlated with overexpression of CCND1, both factors that work closely together in stimulating growth of cancer cells $[33,34]$. When cyclin D1 activates CDKs, it can cause the pRb tumor suppressor protein to become phosphorylated, which releases transcription factors, such as E2F that drive DNA synthesis [35,36]. This increases proliferation, contributing to the pathology of breast cancer. Levels of cyclin D1 are affected by expression by CCND1 and can be used to give patients a more accurate prognosis, as shown in Figure 3. A study involving mice found that mice lacking cyclin D1 did not develop mammary carcinomas even when the ErbB-2 oncogene was activated, which would normally trigger development of cancer cells. These findings, considered with the common overexpression of cyclin D1 in breast cancer in humans, make a compelling case for the significant role of cyclin D1 in breast cancer. Additionally, a study completed from January 2016 to June 2017 involving multiple breast cancer patients found that elevated levels of cyclin D1 was present in $60 \%$ of cases and that the correlation between cyclin D1 and other common markers of breast cancer makes cyclin D1 a favorable prognostic marker of breast cancer.

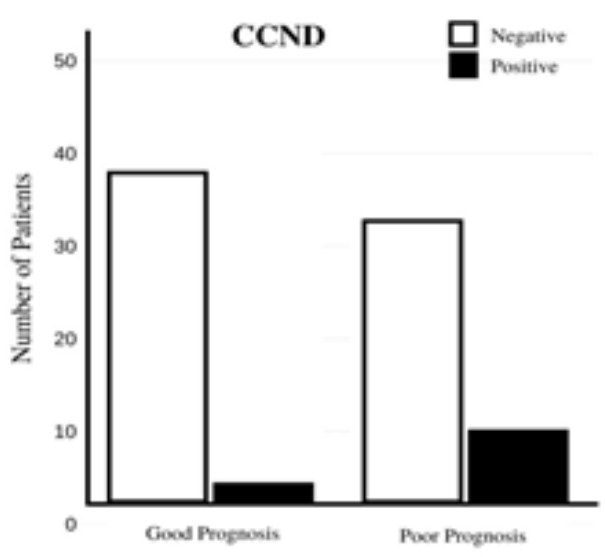

Figure 3: CCND1 can lead to overexpression of cyclin D1, which can be used to give a more accurate prognosis [35].

Another cyclin that can be involved in the development of breast cancer is cyclin E. Though its overexpression is less common than that of cyclin D1, the degradation of its pathways leads to accumulation of other products that impede on the cell cycle. Like cyclin $\mathrm{D} 1$, cyclin $\mathrm{E}$ can phosphorylate $\mathrm{rPb}$, inducing proliferation, but it can also push a cell into $\mathrm{S}$ phase without $\mathrm{rPb}$ or E2F [36]. The two cyclin abnormalities differ in that cyclin D1 overexpression is more common in breast cancer, but cyclin E overexpression leads to breast cancer with higher proliferation rates and worse outcomes [37]. In both cases, overexpression of the cyclin does not correlate with overexpression of the gene, indicating that the problem likely originates from alterations in the degradation pathway [37].

\section{Role in the Metastasis of Breast Cancer}

The leading cause of death in breast cancer is metastasis, or stage IV breast cancer, which is when the previously localized cancer spreads to other areas of the body, most commonly the liver, brain, bones, or lungs [38]. Development of metastatic breast cancer means changes to the treatment of the cancer must be made and be able to match the mobilization of the cancer. Because the tumor cells in the body have traveled away from primary tumor cells, therapies that targeted certain characteristics of primary tumor cells will likely no longer be effective, creating a need for combination therapies that will be able to compensate for these changes [39]. For this reason and others shown in Figure 4, treatments involving CDK inhibitors have been shown to be able to account for and target metastatic cancer cells, even as they continue to spread and proliferate. Similar to abnormalities in the CDK pathways of early stage breast cancer, abnormalities in CDK inhibitors play a role in metastatic breast cancer. The lack of regulation of the cell cycle leads to uncontrolled proliferation, and lack of control of CDKs, which drive the cell cycle, further contribute to this. For this reason, CDK inhibitors can be referred to as potential tumor suppressor genes, although their exact mechanisms are still controversial. However, studies have shown hypermethylation of CDK inhibitors, specifically p16, in primary and metastatic breast cancer cells [40].

\section{Hallmarks of Metastasis and Their Implications for Personalized Medicine}

\begin{tabular}{|c|c|}
\hline Hallmark & Implication \\
\hline $\begin{array}{l}\text { - Heterogeneity between primary } \\
\text { tumors and metastases, and } \\
\text { among metastases }\end{array}$ & $\begin{array}{l}\text { - Therapy based on primary } \\
\text { tumor characteristics may be } \\
\text { ineffective }\end{array}$ \\
\hline $\begin{array}{l}\text { - Redundancy of mechanistic } \\
\text { pathways }\end{array}$ & $\begin{array}{l}\text { - Need for combination } \\
\text { therapies }\end{array}$ \\
\hline - Variable dormancy & $\begin{array}{l}\text { - Clinical trials must address } \\
\text { delayed relapses. }\end{array}$ \\
\hline $\begin{array}{l}\text { - Contribution of cancer- } \\
\text { initiating cells }\end{array}$ & $\begin{array}{l}\text { - Incorporation of therapies that } \\
\text { target stem cells }\end{array}$ \\
\hline
\end{tabular}

Figure 4: Once a cancer has metastasized, treating it becomes complicated. These are different

characteristics of metastasis that have different consequences to consider when developing treatments [39].

The role CDKs play in the metastasis of breast cancer mirror that of their role in early stages of breast cancer. However, because metastatic breast cancer is systemic, the implications differ. As new 
tumor cells spread through the body, abnormalities in cell cycle regulation, such as overexpression of a certain cyclin, continue to induce cell division. Moreover, cyclin D1 is not always involved in directly driving cell division. It can also physically bind to estrogen receptors, which can be devastating to $\mathrm{ER}+$ metastatic breast cancer, as new tumor cells migrate away from primary tumor cells, creating more opportunity for cyclin D1 to bind to estrogen receptors [40]. This also leads to the conclusion that cyclin D1 is regulated by a positive feedback loop, which would mean even higher rates of cell division as tumor cells continue to metastasize; the results of a positive feedback loop in cyclin D1 are demonstrated in Figure 3, which show the overexpression of cyclin D1 in response to CCND1.

The body's response to metastatic breast cancer is significant to the prognosis in each patient because this response can cause growth of smaller disseminated tumors that would have otherwise remained harmless. These tumor cells may have stayed indolent; however, in the presence of carcinogens, like cyclin D1, they could create a systemic response and metastasize [40,41]. This is a reflection of how the dual purpose of cyclins can create larger problems for the body by generating a larger chance of a harmful response; far-reaching consequences of the interactions of CDKs with the cell cycle also encourage more aggressive research in this field. Unfortunately, these can also be difficult to detect as seen with cyclin E, which can stimulate the cell cycle but not detectably overexpressed due to an isoform that binds to CDK2 and remains active throughout the entire cell cycle [40]. Some studies have suggested that cyclin D1-CDK4/6 complexes and cyclin E-CDK2 complexes are often required to work together to maintain a normal cell cycle by inactivating $\mathrm{pRB}$, which reveals the complex nature of how CDKs play a role in the cell cycle, as well as the development of breast cancer.

\section{Advancements in Treatments}

Due to the known role of CDKs in cell division and regulating checkpoints, CDK pathways have long since been a point of interest in treating breast cancer. The hope is to locate the origin of the abnormality in the cell cycle and develop a treatment that will allow cells to regain control of proliferation. Using CDK inhibitors to develop treatments has yielded much promise in the past but, obviously, was not a clean fix. Now with the development of new treatments and combination therapies, new results have shown that the relationship between CDKs and breast cancer has more potential to uncover. In particular, CDK 4/6 and inhibitors of these CDKs have been found to regulate pathways that are significant to the development of tumor cells in breast cancer.

In the cyclin D1-CDK4 pathways, RB is phosphorylated, then hyperphosphorylated by a positive feedback loop involving cyclin $\mathrm{E}$ and the E2F family of transcription factors. Inhibitors of CDK 4/6 are able to stop the phosphorylation of RB, arresting the cell in the G1 phase [42]. Regulating the cyclin D-CDK 4/6-retinoblastoma pathway can be more favorable in some circumstances. For example, cells have endogenous proteins, such as the INK4 proteins, that can inhibit CDK $4 / 6$ activity by binding to the catalytic subunits on CDK $4 / 6$. However, some cells have a deletion of the gene that encodes p16, one of these proteins. This leads to a higher baseline of CDK 4/6 activity, which, theoretically, would make these cells more susceptible to being treated by CDK 4/6 inhibitors [42].

The most commonly diagnosed form of breast cancer is ER+/ HER2- breast cancer; therefore, many treatments are developed to specifically target pathways in this form [43]. Because this form of breast cancer has a higher risk of recurrence, treatments are often less efficient in the long run. CDK 4/6 inhibitors are meant to activate the RB tumor suppressor response, but they have also been shown to improve prognosis in breast cancer for unknown reasons. This could be related to subsets of breast cancers that are not studied as much; nevertheless, these responses to CDK 4/6 inhibition help prevent future recurrences. CDK 4/6 inhibition combined with endocrine therapy has proven to limit the growth of tumor cells, and the two treatments are shown to complement each other. CDK 4/6 inhibitor treatments surprisingly yielded results that showed the induction of genes that may have led to further cell growth. Fortunately, the use of endocrine therapy was shown to block this response, limiting cycling D1 activity [43]. Because one round of growth of tumor cells would lead to another round of growth, this function of endocrine therapy working with CDK 4/6 inhibitors is extremely significant.

Three FDA approved CDK 4/6 inhibitors are palbociclib, ribociclib, and abemaciclib, each designed to work with another form of therapy [44]. Palbociclib, as shown in Figure 5, specifically, was approved to be used in combination with fulvestrant for ER+/HER2- metastatic breast cancer. As mentioned in Figure 4, metastatic cancers often respond relatively well to combination therapies because of their redundancy of mechanical pathways. All three inhibitors were studied in 2017 in a study that found that, due to their low toxicity but high efficiency and oral dosage, these inhibitors were an important option for patients [45]. Palbociclib used in conjunction with endocrine therapy was scrutinized for any updates on efficiency, safety, and pharmacology in 2018; the results showed that this combination therapy improved quality of life and progression-free survival in patients with metastatic breast cancer $[46,47]$.

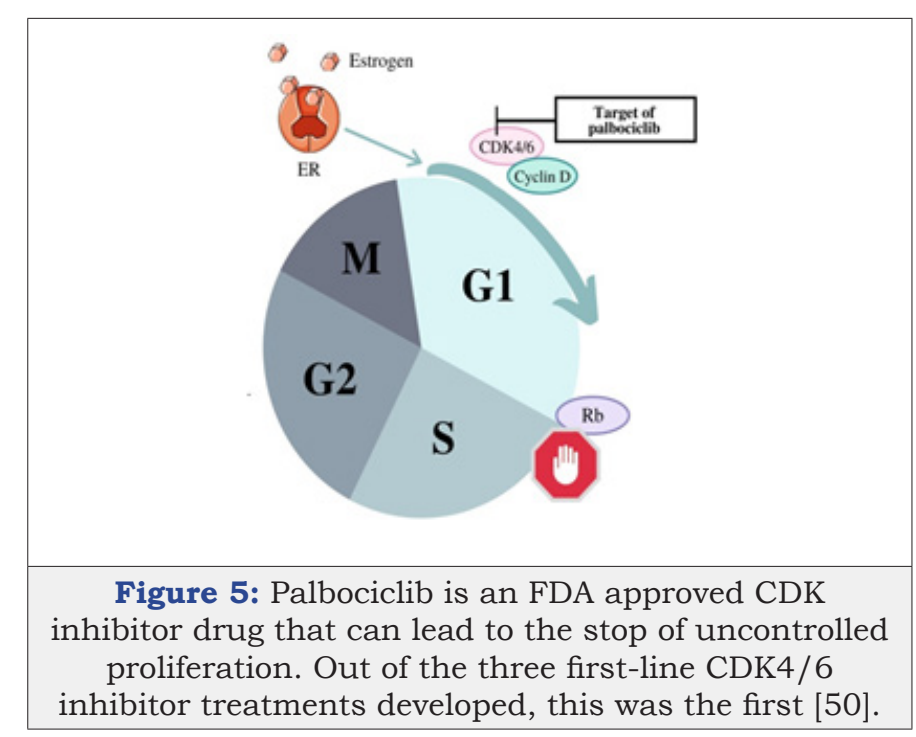


As of 2019, palbociclib was the only CDK4/6 inhibitor whose effects, when studied in clinical settings, yielded results with improved overall survival $[48,49]$. When palbociclib entered phase III trials to be tested with adjuvant endocrine therapy, promising results from phase II testing gave researchers reason to believe in the efficacy of palbociclib. Phase II involved postmenopausal, treatment-naive patients with ER+/HER2metastatic breast cancer who received either only letrozole treatment or a combination treatment of letrozole and palbociclib [49]. Palbociclib monotherapy was shown to have clinical activity; it also demonstrated clinical activity when received with the same endocrine therapy given to patients in moderation prior to the progression of disease [50]. In phase III, researchers continued to test palbociclib with letrozole but also tested it with fulvestrant. A summary of the available clinical data from these trials concluded that the combination of a CDK 4/6 inhibitor, specifically palbociclib as it is the first in the field, with endocrine therapy, either letrozole or fulvestrant, made strides in the management of metastatic breast cancer showing more than a 10-month improvement in median progression-free survival (mPFS), as well as signs of reversing endocrine resistance in patients [49,51]. A study from the Siteman Cancer Center published in 2019 concluded that because ER+/ HER2- breast cancer can be unpredictable due to its molecular and clinical heterogeneity, resistance to CDK 4/6 inhibitors may occur through different mechanisms. Therefore, different patients may need individualized treatment approaches [52]. For example, in a study from 2018, patients who were previously known to have sensitivities to endocrine therapy responded to a combination treatment of palbociclib and fulvestrant with a longer overall survival than the placebo and fulvestrant treatment [53]. As an adjuvant therapy, fulvestrant has been shown to avoid some of the detrimental side effects of selective estrogen receptor modulators like tamoxifen [54].

Like palbociclib, ribociclib is a first-line combination therapy approved for use in the treatment of ER+/HER2- breast cancer, specifically for patients with visceral metastases, bone-only diseases, de novo diseases, and prior therapy [55]. In these subgroups, ribociclib was tested in phase III trials and established itself as a beneficial treatment. Clinical data from these trials also showed that the most common adverse events (AEs) were neutropenia, leukopenia, abnormal liver function tests, infections, and vomiting; still, in testing ribociclib with letrozole, the safety profile of the combination treatment was consistent across all subgroups, and $\mathrm{mPFS}$ and media clinical benefit response ( $\mathrm{mCBR}$ ) were higher in the ribociclib group compared to the placebo group [55]. In the context of combination with endocrine therapy, ribociclib was shown to significantly increase overall survival in a study of 672 patients when received with goserelin and either a nonsteroidal aromatase inhibitor or tamoxifen compared to only endocrine therapy [56]. In the ribociclib group, the estimated overall survival at 42 months was $70.2 \%$ with a $95 \%$ confidence interval (CI) of 63.5 and 76.0 . In the placebo group, estimated overall survival was $46.0 \%$ with a $95 \%$ CI of 32.0 to 58.9 [56]. Regarding the AEs, partly because of CDK4/6 inhibitors ability to be received over long periods of time, the toxicity of both palbociclib and ribociclib have been studied, even in conjunction with each other to further understand drugdrug interactions between them [57]. This research delves into how to manage each of the treatments and their side effects.

The third and most recently developed treatment is abemaciclib, which is a CDK4/6 inhibitor that prevents the progression of the cell cycle by blocking retinoblastoma tumor suppressor protein phosphorylation [58]. Abemaciclib is viewed as a favorable option because it can be received by patients continuously, orally, and as a monotherapy [59]. In clinical trials, abemaciclib response rates ranged from $19.7 \%$ to $59 \%$ with significantly increased mPFS in patients. It also demonstrated similar AEs to both palbociclib and ribociclib [58,59]. As shown in Figure 6, all three treatments have demonstrated neutropenia and leukopenia as AEs but differ in others; for instance, instead of neutropenia forcing lower dosages, fatigue limited the dosages of abemaciclib. Unlike the other two treatments, abemaciclib has a stronger influence in the body, possibly due to its higher specificity for CDK 4 [59]. Results of receiving abemaciclib were seen in MONARCH 2, a phase III clinical trial testing abemaciclib with fulvestrant. Out of the 669 enrolled patients, $25.3 \%$ were determined to have primary endocrine therapy resistance; the group with abemaciclib and fulvestrant had a clinical benefit rate of $72.2 \%$ and mPFS of 16.4 months compared to $56.1 \%$ and 9.3 months in the placebo group [59]. Abemaciclib has also been studied with regards to specific aspects of ER+/ HER2- breast cancer, such as central nervous system metastasis, liver metastasis, and faster tumor shrinkage $[59,60]$. Results of MONARCH 2 also showed that patients who received abemaciclib were able to delay receipt of chemotherapy [61]

\begin{tabular}{|c|c|c|}
\hline Palbociclib & Ribociclib & Abemaciclib \\
\hline Neutropenia & Neutropenia & Neutropenia \\
\hline Leukopenia & Leukopenia & Leukopenia \\
\hline Infections & Abnormal LFTs & Diarrhea \\
\hline Anemia & Vomiting & Anemia \\
\hline
\end{tabular}

Figure 6: Palbociclib, ribociclib, and abemaciclib all have relatively similar adverse events (AEs). These are the most commonly reported grade 3 or 4 toxicities.

All three treatments have had patients show signs of neutropenia and leukopenia [58].

Although there are no head to head trials between the three, given the nature of each drug, patients are able to try another one if the one they have been or had been receiving resulted in intolerance [62]. Although currently, loss of the RB tumor suppressor function is the only indication that the drug is taking clinical effect, further research will look to expand the possible biomarkers for CDK4/6 inhibition [63]. Another possibility researchers have considered is the use of CDK4/6 inhibition before chemotherapy; especially in elderly patients, this has shown signs in clinical trials of reducing treatment-related toxicities caused by chemotherapy [64]. 
Despite the promise shown in combination therapies involving CDK 4/6 inhibitors, endocrine therapy resistance still poses a problem in hormone-sensitive cancers. Especially in metastatic breast cancer, endocrine therapy is designed to target estrogen receptors and stimulation [65]. However, some patients with ER+ breast cancer do not respond to endocrine therapy, which is called de novo resistance, or become resistant to it as the treatment continues, which is called acquired resistance. Because of this, the efficiency of combination therapies can be difficult to gauge; there are more factors at play, and a refractory response to endocrine therapy in the middle of treatment can have negative effects on CDK inhibition-related treatments.

\section{Conclusion}

The study of CDKs in the context of breast cancer and metastatic breast cancer provides much insight into the way the cell cycle is controlled, as well as how the mechanisms of CDK pathways relates to the pathology of breast cancer. Specifically for most commonly occurring forms of breast cancer, such as ER+/HER2- breast cancer, treatments involving CDK pathways are in development as further implications of CDKs and their functions are uncovered. Taking advantage of this relation provides opportunities for development of new treatments, as well as refinement of existing ones. The structure of CDKs gives them the ability to control specific parts of the cell cycle, depending on the cyclin-CDK complex. Extending the depth of understanding around the interconnectedness of the cell cycle, cyclins, and CDKs allows researchers to target specific pathways that have been shown to have abnormalities in the development and metastasis of breast cancer. The cyclin and CDK pairing also creates dependencies in cell division that highlight the integral role that CDKs play in mitosis. CDKs are able to both directly and indirectly influence the cell cycle, emphasizing the importance of their integrity in preventing uncontrolled cell proliferation. In the development of breast cancer, abnormalities in the pathways of cyclin E and cyclin D1 have been found to play a role; these two cyclins are also linked specifically to estrogen receptor positive breast cancer. Cyclin D1's ability to act as a carcinogen also affects the diagnosis and development of breast cancer, acting as a marker that something is abnormal in the body. Furthermore, the distinct ways cyclin D1 and cyclin E behave in breast cancer allows them to act as markers for different prognoses. Because of this, they are both targets in the topic of using CDK inhibition to treat advanced breast cancer. Other areas of the cell, such as pRB and CCND1, have been implicated in the response caused by abnormal levels of cyclin D1 and cyclin E. With this knowledge on CDKs, advancements in treatments have been made revolving around CDK inhibition. Three FDA approved CDK 4/6 inhibitors are palbociclib, ribociclib, and abemaciclib; each of these treatments have been tested as monotherapies and with adjuvant endocrine therapy, such as fulvestrant and letrozole. Promising outcomes encourage future use of these for the treatment of ER+/HER2- metastatic breast cancer. CDK4/6 inhibition has shown in a clinical setting to be well-tolerated and efficient through increased overall survival compared to placebo groups and elevated mPFS and mCBR. While the results of using these treatments on ER+ breast cancer show obvious benefits over other more detrimental treatments like chemotherapy and radiation treatments, there are still obstacles to overcome, such as endocrine therapy resistance. Adverse events that consistently appear in patients receiving these treatments, including neutropenia and fatigue, are often able to be treated symptomatically and without further complication. Future studies into this area of research aim to refine and improve the use of CDK inhibitors to treat breast cancer.

\section{Conflict of Interest}

We have no conflicts of interest to declare.

\section{Acknowledgment}

We thank Ava Wang for generating the figures used in this paper.

\section{References}

1. Noble M, Endicott J (2000) Cyclin-dependent kinases.

2. Johnson ES, Kornbluth SS (2012) Phosphatases driving mitosis: Pushing the gas and lifting the brakes. Prog Mol Biol Transl Sci 106: 327-341.

3. Rahal R, Amon A(2008) Mitotic CDKs control the metaphase-anaphase transition and trigger spindle elongation. Genes \& Development 22(11): 1534-1548.

4. Nurse P, Thuriaux P (19) Regulatory genes controlling mitosis in the fission yeast schizosaccharomyces pombe. Genetics 96(3): 627-637.

5. Cooper GM (2000) The cell: A molecular approach. ( $2^{\text {nd }}$ edn), The Development and Causes of Cancer, Sunderland (MA): Sinauer Associates, USA.

6. Carrassa L (2014) Cell cycle, checkpoints, cancer. Atlas Genet Cytogenet Oncol Haematol 18(1): 67-75.

7. Richard S Finn, Alexey Aleshin, Dennis J Slamon (2016) Targeting the cyclin-dependent kinases (CDK) 4/6 in estrogen receptor-positive breast cancers. Breast Cancer Res 18(1): 17.

8. Davis ST, Benson BG, Bramson HN, Chapman DE, Dickerson SH, et al. (2001) Prevention of chemotherapy-induced alopecia in rats by cdk inhibitors. Science 291(5501): 134-137.

9. Venur VA, Leone JP (2016) Targeted therapies for brain metastases from breast cancer. International Journal of Molecular Sciences 17(9): 1543.

10. Bossemeyer D (1995) Protein kinases--structure and function. FEBS Lett 369(1): 57-61.

11. Pavletich NP (1999) Mechanisms of cyclin-dependent kinase regulation: Structures of cdks, their cyclin activators, and cip and INK4 inhibitors. J Mol Biol 287(5): 821-828.

12. Manning G. Protein Kinases: Introduction. Cell Signaling Technology.

13. Morgan DO (1997) Cyclin-Dependent Kinases: Engines, clocks, and microprocessors. Annu Rev Cell Dev Biol 13: 261-291.

14. Bondt HL, Rosenblatt J, Jancarik J, Jones HD, Morgant DO, et al. (1993) Crystal structure of cyclin-dependent kinase 2. Nature 363(6430): 595602.

15. Darzynkiewicz Z, Gong J, Juan G, Ardelt B, Traganos F (1996) Cytometry of cyclin proteins. Cytometry 25(1): 1-13.

16. CDK . Scitable by Nature Education.

17. Suryadinata R, Sadowski M, Sarcevic B (2010) Control of cell cycle progression by phosphorylation of cyclin-dependent kinase (CDK) substrates. Biosci Rep 30(4): 243-255.

18. Lim S, Kaldis P (2013) Cdks, cyclins and CKIs: Roles beyond cell cycle regulation. Development 140(15): 3079-3093. 
19. Barnum KJ, Connell MJ (2014) Cell cycle regulation by checkpoints Methods in Molecular Biology 1170: 29-40.

20. Wenzel ES, Singh A (2018) Cell-cycle checkpoints and aneuploidy on the path to cancer. In vivo 32(1): 1-5.

21. Fishman DD, Segal S, Livneh E (1998) The role of protein kinase C in G1 and G2/M phases of the cell cycle (review). Int J Oncol 12(1):181-186.

22. Fisher RP (2016) Getting to S: CDK functions and targets on the path to cell-cycle commitment. F1000 Research 5: 2374.

23. Solaki M, Ewald JC (2018) Fueling the cycle: CDKs in carbon and energy metabolism. Front Cell Dev Biol 6:93.

24. Blanchet E, Annicotte JS, Fajas L (2009) Cell cycle regulators in the control of metabolism. Cell Cycle 8(24): 4029-4031.

25. Loyer P, Trembley JH, Katona R, Kidd VJ, Lahti JM (2005) Role of CDK/ cyclin complexes. Cell Signal 17(9): 1033-1051.

26. Alberts B, Johnson A, Lewis J, et al. (2002) Molecular biology of the cell. $\left(4^{\text {th }}\right.$ edn), Intracellular Control of Cell-Cycle Events, Garland Science, New York, USA.

27. Cheng A, Ross KE, Kaldis P, Solomon MJ (1999) Dephosphorylation of cyclin-dependent kinases by type 2C protein phosphatases. Genes \& Development 13(22): 2946-2957.

28. AM, MH T. Biological profile of eruct: A new promising anticancer agent from cruciferous vegetables.

29. Feng Y, Spezia M, Huang S, Yuan C, Zeng Z, et al. (2018) Breast cancer development and progression: Risk factors, cancer stem cells, signaling pathways, genomics, and molecular pathogenesis. Genes \& Diseases 5(2): 77-106

30. Bashour SI, Doostan I, Keyomarsi K, Valero V, Ueno NT, et al. (2017) Rapid breast cancer disease progression following cyclin dependent kinase 4 and 6 inhibitor discontinuation. J Cancer 8(11): 2004-2009.

31. Breast Cancer Hormone Receptor Status: Estrogen Receptor.

32. Murphy CG, Dickler MN (2015) The role of CDK4/6 inhibition in breast cancer. The Oncologist 20(5): 483-490.

33. Mohammadizadeh F, Hani M, Ranaee M, Bagheri M (2013) Role of cyclin D1 in breast carcinoma. Journal of Research in Medical Sciences 18(12): 1021-1025.

34. Eeckhoute J, Carroll JS, Geistlinger TR, Torres-Arzayus MI, Brown M (2006) A cell-type-specific transcriptional network required for estrogen regulation of cyclin D1 and cell cycle progression in breast cancer. Genes \& Development 20(18): 2513-2526. 1058.

35. He J, Wang H, Ma F, Feng F, Lin C, et al. (2014) Prognosis of lymph nodenegative breast cancer: Association with clinicopathological factors and tumor associated gene expression. Oncol Lett 8(4): 1717-1724.

36. Osborne C, Wilson P, Tripathy D (2004) Oncogenes and tumor suppressor genes in breast cancer: potential diagnostic and therapeutic applications. Oncologist 9(4): 361-377.

37. Lodén M, Stighall M, Nielsen NH, Roos G, Emdin SO, et al. (2002)The cyclin D1 high and cyclin E high subgroups of breast cancer: Separate pathways in tumorogenesis based on pattern of genetic aberrations and inactivation of the pRb node. Oncogene 21(30): 4680-4690.

38. Metastatic Breast Cancer: Symptoms, Treatment, and More.

39. Marino N, Woditschka S, Reed LT, Nakayama J, Mayer M, et al. (2013) Breast cancer metastasis: Issues for the personalization of its prevention and treatment. The American Journal of Pathology 183(4): 1084-1095.

40. Fernandez P, Jares P, Rey M, Campo E, Cardesa A (1998) Cell cycle regulators and their abnormalities in breast cancer. Mol Pathol 51(6): 305-309.

41. Redig AJ, McAllister SS (2013) Breast cancer as a systemic disease: A view of metastasis. Journal of Internal Medicine 274(2): 113-126.
42. Pernas S, Tolaney SM, Winer EP, Goel S (2018) CDK4/6 inhibition in breast cancer: Current practice and future directions. Therapeutic Advances in Medical Oncology 10: 1758835918786451.

43. Knudsen ES, Witkiewicz AK (2016) Defining the transcriptional and biological response to CDK4/6 inhibition in relation to ER+/HER2breast cancer. Oncotarget 7(43): 69111-69123.

44. Columbus G. CDK 4/6 Inhibitors revolutionized breast cancer treatment.

45. Kwapisz D (2017) Cyclin-dependent kinase 4/6 inhibitors in breast cancer: Palbociclib, ribociclib, and abemaciclib. Breast Cancer Res Treat 166(1): 41-54.

46. Yu Q, Sicinska E, Geng Y, Ahnström M, Zagozdzon A (2006) Requirement for CDK4 kinase function in breast cancer. Cancer Cell 9(1): 23-32.

47. McShane TM, Wolfe TA, Ryann JC (2018) Updates on managing advanced breast cancer with palbociclib combination therapy. Ther Adv Med Oncol 10: 1758835918793849.

48. Parvin T, Das C, Choudhury M, Chattopadhyay BK, Mukhopadhyay M (2019) Prognostic utility of cyclin D1 in invasive breast carcinoma. Indian J Surg Oncol 10(1): 167-173.

49. Serra F, Lapidari P, Quaquarini E, Tagliaferri B, Sottotetti F, et al. (2019) Palbociclib in metastatic breast cancer: Current evidence and real-life data. Drugs in Context 8: 212579.

50. Orbaugh K, Ryan JC, Pfeuffer L (2016) Palbociclib Plus letrozole for the treatment of metastatic breast cancer: An illustrative case scenario. Journal of the Advanced Practitioner in Oncology 7(5): 550-561.

51. Malorni L, Curigliano G, Minisini AM, Cinieri S, Tondini CA, et al. (2018) Palbociclib as single agent or in combination with the endocrine therapy received before disease progression for estrogen receptorpositive, HER2-negative metastatic breast cancer: TREnd trial. Annals of Oncology 29(8): 1748-1754.

52.Xi J, Oza A, Thomas S, Ademuyiwa F, Weilbaecher K, et al. (2019) Retrospective analysis of treatment patterns and effectiveness of palbociclib and subsequent regimens in metastatic breast cancer. Journal of the National Comprehensive Cancer Network 17(2): 141-147.

53. Turner NC, Slamon DJ, Ro J, Bondarenko I, Im SA, et al. (2018) Overall survival with palbociclib and fulvestrant in advanced breast Cancer. N Engl J Med 379(20): 1926-1936.

54. Johnston SJ, Cheung KL (2010) Fulvestrant - A novel endocrine therapy for breast cancer. Current Medicinal Chemistry 17(10): 902-914.

55. Hortobagyi GN (2018) Ribociclib for the first-line treatment of advanced hormone receptor-positive breast cancer: a review of subgroup analyses from the MONALEESA-2 trial. Breast Cancer Research 20(1): 123.

56. Im SA, Lu YS, Bardia A, Harbeck N, Colleoni M, et al. (2019) Overall survival with ribociclib plus endocrine therapy in breast cancer. $\mathrm{N}$ Engl J Med 381(4): 307-316.

57. Bellet M, Ahmad F, Villanueva R, Valdivia C, Palomino-Doza J, et al. (2019) Palbociclib and ribociclib in breast cancer: consensus workshop on the management of concomitant medication. Therapeutic Advances in Medical Oncology 11: 1758835919833867.

58. Palumbo A, Lau G, Saraceni M (2019) Abemaciclib: The newest CDK4/6 inhibitor for the treatment of breast cancer. Ann Pharmacother 53(2):178-185.

59. Martin JM, Goldstein LJ (2018) Profile of abemaciclib and its potential in the treatment of breast cancer. Onco Targets and Therapy 11: 52535259.

60. Kenji Tamura (2019) Differences of cyclin-dependent kinase 4/6 inhibitor, palbociclib and abemaciclib, in breast cancer. Japanese Journal of Clinical Oncology 49(11): 993-998.

61. Sledge GW, Toi M, Neven P, Sohn J, Inoue K, et al. (2019) The effect of abemaciclib plus fulvestrant on overall survival in hormone receptorpositive, ERBB2-negative breast cancer that progressed on endocrine 
therapy-MONARCH 2: A randomized clinical trial. JAMA Oncology 6(1): 116-124.

62. Mohammed AA, Rashied H, Elsayed FM (2019) CDK4/6 inhibitors in advanced breast cancer, what is beyond? Oncology Reviews 13(2): 416.

63. Knudsen ES, Witkiewicz AK (2017) The strange case of CDK4/6 inhibitors: Mechanisms, resistance, and combination strategies. Trends in Cancer 3(1): 39-55.
64. Matutino A, Amaro C, Verma S (2018) CDK4/6 inhibitors in breast cancer: Beyond hormone receptor-positive HER2-negative disease. Therapeutic Advances in Medical Oncology 10: 1758835918818346.

65. Fan W, Chang J, Fu P (2015) Endocrine therapy resistance in breast cancer: current status, possible mechanisms and overcoming strategies. Future Medicinal Chemistry 7(12): 1511-1519. 